\title{
GOODNESS-OF-FIT TESTS BASED \\ ON CHARACTERIZATIONS OF CONTINUOUS DISTRIBUTIONS
}

Abstract. We construct goodness-of-fit tests for continuous distributions using their characterizations in terms of moments of order statistics and moments of record values. Our approach is based on characterizations presented in [2]-[4], [5], [9].

1. Introduction. Let $\left(X_{1}, \ldots, X_{n}\right)$ be a random sample from a distribution $F(x)=P[X \leq x], x \in \mathbb{R}$, and let $X_{k: n}$ denote the $k$ th smallest order statistic of the sample. In what follows we use the following characterizations of continuous distributions via moments of functions of order statistics.

Theorem 1 (cf. [9]). Let $m$ be a positive integer and $E X_{k: n}^{2}<\infty$ for some pair $(k, n)$. Then

$$
\frac{(k-1) !}{n !} E X_{k: n}^{2}-2 \frac{(k+m-1) !}{(n+m) !} E X_{k+m: n+m}+\frac{(k+2 m-1) !}{(n+2 m) !}=0
$$

iff $F(x)=x^{1 / m}$ on $(0,1)$.

Taking $k=n=1$, we get

Corollary 1. $F(x)=x^{1 / m}$ on $(0,1)$ iff

$$
\frac{2}{m+1} E X_{m+1: m+1}-E X^{2}=\frac{1}{2 m+1} \text {. }
$$

In particular, $X \sim U(0,1)$ iff $E X_{2: 2}-E X^{2}=1 / 3$.

2000 Mathematics Subject Classification: Primary 62E10, 62F03.

Key words and phrases: order statistics; $k$-record values; characterization of distributions; uniform, Weibull, exponential, Pareto distributions; goodness-of-fit tests; significance probability. 
In the following theorems, $X$ denotes a random variable with distribution $F$, and $I(F)$ denotes the minimal interval containing the support of $F$.

TheOrem 2 (cf. [5]). Let $n, k, l$ be given integers such that $n \geq k \geq l \geq$ 1. Assume that $G$ is a nondecreasing right-continuous function from $\mathbb{R}$ to $\mathbb{R}$. Then the relations

$$
\begin{aligned}
& E G^{l}\left(X_{k+1: n+1}\right)=\frac{(k+1) \ldots(k+l)}{(n+2) \ldots(n+l+1)}, \\
& E G^{2 l}\left(X_{k+1-l: n+1-l}\right)=\frac{(k-l+1) \ldots(k+l)}{(n-l+2) \ldots(n+l+1)}
\end{aligned}
$$

hold iff $F(x)=G(x)$ on $I(F)$ and $F$ is continuous on $\mathbb{R}$.

Taking $n=k=l=1$, we get

Corollary 2. $F(x)=G(x)$ on $I(F)$ and $F$ is continuous on $\mathbb{R}$ iff $E G^{2}(X)=1 / 3$ and $E G\left(X_{2: 2}\right)=2 / 3$.

TheOrem 3 (cf. [2], [3]). Under the assumptions of Theorem 2, $F(x)=$ $G(x)$ on $I(F)$ and $F$ is continuous on $\mathbb{R}$ iff

$$
\begin{aligned}
& \frac{(k-l) !}{(n-l+1) !} E G^{2 l}\left(X_{k+1-l: n+1-l}\right) \\
& -\frac{2 k !}{(n+1) !} E G^{l}\left(X_{k+1: n+1}\right)+\frac{(k+l) !}{(n+l+1) !}=0 .
\end{aligned}
$$

Taking $n=k=l=1$, we get

COROllary 3. $F(x)=G(x)$ on $I(F)$ and $F$ is continuous on $\mathbb{R}$ iff

$$
E G\left(X_{2: 2}\right)-E G^{2}(X)=1 / 3 .
$$

Before quoting characterization theorems in terms of moments of record values we give the definition of $k$-record values (cf. [1]).

Let $\left\{X_{n}, n \geq 1\right\}$ be a sequence of i.i.d. random variables with $\operatorname{cdf} F$ and pdf $f$. For a fixed $k \geq 1$ we define the sequence $U_{k}(1), U_{k}(2), \ldots$ of $k$-(upper) record times of $X_{1}, X_{2}, \ldots$ as follows: $U_{k}(1)=1$, and for $n=2,3, \ldots$,

$$
U_{k}(n)=\min \left\{j>U_{k}(n-1): X_{j: j+k-1}>X_{U_{k}(n-1): U_{k}(n-1)+k-1}\right\} .
$$

Write

$$
Y_{n}^{(k)}:=X_{U_{k}(n): U_{k}(n)+k-1}, \quad n \geq 1 .
$$

The sequence $\left\{Y_{n}^{(k)}, n \geq 1\right\}$ is called the sequence of $k$-(upper) record values of the above sequence. For convenience we also take $Y_{0}^{(k)}=0$ and note that $Y_{1}^{(k)}=X_{1: k}=\min \left(X_{1}, \ldots, X_{k}\right)$.

We shall apply the following characterization results:

TheOrem 4 (cf. [3], [4]). Let $\left\{X_{n}, n \geq 1\right\}$ be a sequence of i.i.d. random variables with cdf $F$. Assume that $G$ is a nondecreasing right-continuous 
function from $\mathbb{R}$ to $(-\infty, 1]$, and let $n, k, l$ be given integers such that $k \geq 1$ and $n \geq l \geq 1$. Then $F(x)=G(x)$ on $I(F)$ iff the following relations hold:

$$
\begin{aligned}
& E\left[-\log \left(1-G\left(Y_{n+1}^{(k)}\right)\right)\right]^{l}=\frac{(n+l) !}{n ! k !}, \\
& E\left[-\log \left(1-G\left(Y_{n-l}^{(k)}\right)\right)\right]^{2 l}=\frac{(n+l) !}{(n-l) ! k^{2 l}} .
\end{aligned}
$$

TheOREm $4^{\prime}$ (cf. [3], [4]). Under the assumptions of Theorem $4, F(x)=$ $G(x)$ on $I(F)$ iff

$$
k^{2 l}(n-l) ! E H_{l}^{2}\left(Y_{n-l+1}^{(k)}\right)-2 n ! k^{l} E H_{l}\left(Y_{n+1}^{(k)}\right)+(n+l) !=0,
$$

where $H_{l}(x)=(-\log (1-G(x)))^{l}, x \in \mathbb{R}$.

In particular $X$ has df $F$ iff

$$
E\left[-\log \left(1-F\left(Y_{1}^{(k)}\right)\right)\right]^{2}-\frac{2}{k} E\left[-\log \left(1-F\left(Y_{2}^{(k)}\right)\right)\right]+\frac{2}{k^{2}}=0 .
$$

Corollary 4. (a) $F(x)=x^{\alpha}$ on $(0,1), \alpha>0$, iff

$$
E\left(-\log \left(1-\left(Y_{1}^{(k)}\right)^{\alpha}\right)\right)^{2}-\frac{2}{k} E\left(-\log \left(1-\left(Y_{2}^{(k)}\right)^{\alpha}\right)\right)+\frac{2}{k^{2}}=0 .
$$

In particular, $X \sim U(0,1)$ iff

$$
E\left(-\log \left(1-Y_{1}^{(k)}\right)\right)^{2}-\frac{2}{k} E\left(-\log \left(1-Y_{2}^{(k)}\right)\right)+\frac{2}{k^{2}}=0 .
$$

(b) $F(x)=1-e^{-(1 / \lambda) x^{\alpha}}, x>0, \alpha>0, \lambda>0$, iff

$$
E\left(Y_{1}^{(k)}\right)^{2 \alpha}-\frac{2 \lambda}{k} E\left(Y_{2}^{(k)}\right)^{\alpha}+\frac{2 \lambda^{2}}{k^{2}}=0 .
$$

In particular, $X \sim \operatorname{Exp}(1 / \lambda)$, i.e. $F(x)=1-e^{-x / \lambda}$, iff

$$
E\left(Y_{1}^{(k)}\right)^{2}-\frac{2 \lambda}{k} E Y_{2}^{(k)}+\frac{2 \lambda^{2}}{k^{2}}=0 .
$$

(c) $F(x)=1-\left(x_{0} / x\right)^{a}, x>x_{0}, a>0$, iff

$$
E\left[-\log \left(\frac{x_{0}}{Y_{1}^{(k)}}\right)\right]^{2}-\frac{2}{k a} E\left[-\log \left(\frac{x_{0}}{Y_{2}^{(k)}}\right)\right]+\frac{2}{k^{2} a^{2}}=0 .
$$

2. Goodness-of-fit tests based on characterizations via moments of order statistics. First note that (1.1) can be written in the form

$$
E\left(F\left(X_{2: 2}\right)\right)-\frac{1}{2}\left(E\left(F^{2}\left(X_{1}\right)\right)+E\left(F^{2}\left(X_{2}\right)\right)\right)=\frac{1}{3}
$$

as $X_{1}$ and $X_{2}$ are distributed as $X$. 
Let $\left(X_{1}, \ldots, X_{2 n}\right)$ be a sample. Write

$$
\begin{aligned}
Y_{j} & =F^{2}\left(X_{2 j-1}\right)+F^{2}\left(X_{2 j}\right), \\
Z_{j} & =F\left(\max \left(X_{2 j-1}, X_{2 j}\right)\right), \quad j=1, \ldots, n .
\end{aligned}
$$

Letting $Y:=Y_{1}=F^{2}\left(X_{1}\right)+F^{2}\left(X_{2}\right), Z:=Z_{1}=F\left(\max \left(X_{1}, X_{2}\right)\right)$, we quote the following result (cf. [6]).

Lemma 1. Under the above assumptions, the density of $(Y, Z)$ is given by

$$
f(y, z)= \begin{cases}1 / \sqrt{y-z^{2}}, & 0 \leq y \leq 2,0 \leq z \leq 1, z^{2} \leq y \leq 2 z^{2} \\ 0, & \text { otherwise }\end{cases}
$$

and

$$
\begin{aligned}
& E Y=2 / 3, \quad \operatorname{Var}(Y)=8 / 45, \\
& E Z=2 / 3, \quad \operatorname{Var}(Z)=1 / 18, \quad \operatorname{Cov}(Y, Z)=4 / 45 .
\end{aligned}
$$

Put

$$
D_{j}=Z_{j}-\frac{1}{2} Y_{j}, \quad j=1, \ldots, n
$$

We see that

$$
E D_{j}=E Z_{j}-\frac{1}{2} E Y_{j}=\frac{1}{3}
$$

$\operatorname{Var} D_{j}=\operatorname{Var} Z_{j}+\frac{1}{4} \operatorname{Var} Y_{j}-\operatorname{Cov}\left(Z_{j}, Y_{j}\right)=\frac{1}{90}, \quad j=1, \ldots, n$.

Now define

$$
V_{n}=3 \sqrt{10 n}\left(\overline{D_{n}}-1 / 3\right),
$$

where $\overline{D_{n}}=(1 / n) \sum_{j=1}^{n} D_{j}$.

Setting $X_{j}^{*}=\max \left(X_{2 j-1}, X_{2 j}\right), j=1, \ldots, n$, we note that $V_{n}$ can be written as

$$
V_{n}=3 \sqrt{10 n}\left(\frac{1}{n} \sum_{j=1}^{n} F\left(X_{j}^{*}\right)-\frac{1}{2 n} \sum_{j=1}^{2 n} F^{2}\left(X_{j}\right)-\frac{1}{3}\right) .
$$

Taking into account that

$$
X_{j}^{*}=\left(X_{2 j-1}+X_{2 j}\right) / 2+\left|X_{2 j}-X_{2 j-1}\right| / 2
$$

and writing

$$
X_{j}^{0}=\left(X_{2 j-1}+X_{2 j}\right) / 2, \quad X_{j}^{+}=\left|X_{2 j}-X_{2 j-1}\right| / 2
$$

we obtain

$$
V_{n}=3 \sqrt{10 n}\left(\left(\overline{F\left(X_{n}^{0}+X_{n}^{+}\right)}-\overline{F^{2}\left(X_{2 n}\right)}\right)-1 / 3\right),
$$


where

$$
\begin{aligned}
\overline{F\left(X_{n}^{0}+X_{n}^{+}\right)} & =\frac{1}{n} \sum_{j=1}^{n} F\left(X_{j}^{0}+X_{j}^{+}\right), \\
\overline{F^{2}\left(X_{2 n}\right)} & =\frac{1}{2 n} \sum_{j=1}^{2 n} F^{2}\left(X_{j}\right) .
\end{aligned}
$$

Moreover, we conclude from the CLT that

$$
V_{n} \stackrel{D}{\rightarrow} V \sim N(0,1)
$$

and hence that

$$
V_{n}^{2} \stackrel{D}{\rightarrow} \chi^{2}(1)
$$

which provides a simple asymptotic test of the hypothesis $X \sim F$ when the parameters of $F$ are specified.

Special cases:

(a) If $F(x)=x^{1 / m}, x \in(0,1), m$ is a positive integer, then

$$
\begin{aligned}
V_{n}=3 \sqrt{10 n}\left(\frac{1}{n} \sum_{j=1}^{n}\left(\left(X_{2 j}+X_{2 j-1}+\left|X_{2 j}-X_{2 j-1}\right|\right) / 2\right)^{1 / m}\right. & \\
& \left.-\frac{1}{2 n} \sum_{j=1}^{2 n} X_{j}^{2 / m}-\frac{1}{3}\right) .
\end{aligned}
$$

In particular, for $X \sim U(0,1)$,

$$
V_{n}=3 \sqrt{10 n}\left(\overline{X_{2 n}}+\overline{X_{n}^{+}}-\overline{X_{2 n}^{2}}-1 / 3\right) .
$$

(b) If $F(x)=x / \beta, x \in(0, \beta), \beta>0$, then

$$
V_{n}=\frac{3 \sqrt{10 n}}{\beta}\left(\overline{X_{2 n}}+\overline{X_{n}^{+}}-\frac{1}{\beta} \overline{X_{2 n}^{2}}-\frac{\beta}{3}\right),
$$

(c) If $F(x)=\frac{x-\alpha}{\beta-\alpha}, x \in(\alpha, \beta)$, then

$$
V_{n}=\frac{3 \sqrt{10 n}}{\beta-\alpha}\left(\frac{\beta+\alpha}{\beta-\alpha} \overline{X_{2 n}}+\overline{X_{n}^{+}}-\frac{1}{\beta-\alpha} \overline{X_{2 n}^{2}}-\frac{\alpha \beta}{\beta-\alpha}-\frac{\beta-\alpha}{3}\right) .
$$

(d) If $F(x)=1-e^{-(1 / \lambda) x^{\alpha}}, x \geq 0, \alpha>0, \lambda>0$, then

$$
\begin{aligned}
V_{n}= & 3 \sqrt{10 n}\left(\frac{1}{n} \sum_{j=1}^{n}\left(1-\exp \left(-\left(X_{2 j}+X_{2 j-1}+\left|X_{2 j}-X_{2 j-1}\right|\right)^{\alpha} /\left(2^{\alpha} \lambda\right)\right)\right)\right. \\
& \left.-\frac{1}{2 n} \sum_{j=1}^{2 n}\left(1-\exp \left(-X_{j}^{\alpha} / \lambda\right)\right)^{2}-\frac{1}{3}\right) .
\end{aligned}
$$


In particular, for $X \sim \operatorname{Exp}(1 / \lambda)$,

$$
\begin{aligned}
V_{n}= & 3 \sqrt{10 n}\left(\frac{1}{n} \sum_{j=1}^{n}\left(1-\exp \left(-\left(X_{2 j}+X_{2 j-1}+\left|X_{2 j}-X_{2 j-1}\right|\right) /(2 \lambda)\right)\right)\right. \\
& \left.-\frac{1}{2 n} \sum_{j=1}^{2 n}\left(1-\exp \left(-X_{j} / \lambda\right)\right)^{2}-\frac{1}{3}\right),
\end{aligned}
$$

(e) If $F(x)=1-\left(x_{0} / x\right)^{a}, x \geq x_{0}, a>0$, then

$$
\begin{aligned}
V_{n}= & 3 \sqrt{10 n}\left(\frac{1}{n} \sum_{j=1}^{n}\left(1-\left(\frac{2 x_{0}}{X_{2 j-1}+X_{2 j}+\left|X_{2 j}-X_{2 j-1}\right|}\right)^{a}\right)\right. \\
& \left.-\frac{1}{2 n} \sum_{j=1}^{2 n}\left(1-\left(\frac{x_{0}}{X_{j}}\right)^{a}\right)^{2}-\frac{1}{3}\right) .
\end{aligned}
$$

From (2.1) we see that in each special case $V_{n}$ converges weakly to the standard normal distribution, and so provides an asymptotic test of the hypothesis $H$ that $X$ has df $F$ in the case when the parameter values are specified by $H$. When $H$ does not specify the parameter values we consider the test statistic obtained from $V_{n}$ by replacing the parameters by estimators. In this case we have the following results.

Proposition 1. When $F(x)=x / \beta, x \in(0, \beta), \beta>0$, the resulting test statistic is

$$
V_{n}\left(\widehat{\beta}_{n}\right):=\frac{3 \sqrt{10 n}}{\widehat{\beta}_{n}}\left(\overline{X_{2 n}}+\overline{X_{n}^{+}}-\frac{1}{\widehat{\beta}_{n}} \overline{X_{2 n}^{2}}-\frac{\widehat{\beta}_{n}}{3}\right) \stackrel{D}{\rightarrow} V \sim N(0,1),
$$

where $\widehat{\beta}_{n}=\max \left(X_{1}, \ldots, X_{2 n}\right)$.

Proof. We write

$$
\begin{aligned}
V_{n}\left(\widehat{\beta}_{n}\right)= & \frac{\beta}{\widehat{\beta}_{n}}\left(\frac{3 \sqrt{10 n}}{\beta}\left(\overline{X_{2 n}}+\overline{X_{n}^{+}}-\frac{1}{\beta} \overline{X_{2 n}^{2}}-\frac{\beta}{3}\right)\right) \\
& -\frac{3 \sqrt{10 n}}{\widehat{\beta}_{n}}\left(\frac{1}{\widehat{\beta}_{n}}-\frac{1}{\beta}\right) \overline{X_{2 n}^{2}}-\frac{\sqrt{10 n}}{\widehat{\beta}_{n}}\left(\widehat{\beta}_{n}-\beta\right) .
\end{aligned}
$$

Note that

$$
-\frac{\sqrt{10 n}}{\widehat{\beta}_{n}}\left(\frac{1}{\widehat{\beta}_{n}}-\frac{1}{\beta}\right) \overline{X_{2 n}^{2}}=\frac{\sqrt{10 n}\left(\widehat{\beta}_{n}-\beta\right)}{\widehat{\beta}_{n}^{2} \beta} \overline{X_{2 n}^{2}} \stackrel{P}{\rightarrow} 0,
$$

as

$$
\overline{X_{2 n}^{2}} \stackrel{P}{\rightarrow} E X^{2} \quad \text { and } \quad 2 n\left(\beta-\widehat{\beta}_{n}\right) \stackrel{D}{\rightarrow} W \sim \operatorname{Exp}(1 / \beta)
$$

The assertion then follows from Slutsky's theorem. 

tic is

Proposition 2. When $F(x)=\frac{x-\alpha}{\beta-\alpha}, x \in(\alpha, \beta)$, the resulting test statis-

$$
\begin{aligned}
V_{n}\left(\widehat{\alpha}_{n}, \widehat{\beta}_{n}\right)= & \frac{3 \sqrt{10 n}}{\widehat{\beta}_{n}-\widehat{\alpha}_{n}}\left(\frac{\widehat{\beta}_{n}+\widehat{\alpha}_{n}}{\widehat{\beta}_{n}-\widehat{\alpha}_{n}} \overline{X_{2 n}}+\overline{X_{n}^{+}}-\frac{1}{\widehat{\beta}_{n}-\widehat{\alpha}_{n}} \overline{X_{2 n}^{2}}\right. \\
& \left.-\frac{\widehat{\alpha}_{n} \widehat{\beta}_{n}}{\widehat{\beta}_{n}-\widehat{\alpha}_{n}}-\frac{\widehat{\beta}_{n}-\widehat{\alpha}_{n}}{3}\right) \stackrel{D}{\rightarrow} V \sim N(0,1),
\end{aligned}
$$

where $\widehat{\beta}_{n}=\max \left(X_{1}, \ldots, X_{2 n}\right)$ and $\widehat{\alpha}_{n}=\min \left(X_{1}, \ldots, X_{2 n}\right)$.

Proof. The proof is similar to the proof of Proposition 1, since

$$
\begin{aligned}
& 2 n\left(\widehat{\alpha}_{n}-\alpha\right) \stackrel{D}{\rightarrow} W_{1} \sim \operatorname{Exp}(1 / \alpha), \quad 2 n\left(\beta-\widehat{\beta}_{n}\right) \stackrel{D}{\rightarrow} W_{2} \sim \operatorname{Exp}(1 / \beta), \\
& X_{2 n} \stackrel{P}{\rightarrow} E X, \quad \overline{X_{2 n}^{2}} \stackrel{P}{\rightarrow} E X^{2} .
\end{aligned}
$$

REMARK. From the above proof we see that one can use estimators $\widehat{\alpha}_{n}$ and $\widehat{\beta}_{n}$ such that

$$
\sqrt{n}\left(\widehat{\alpha}_{n}-\alpha\right) \stackrel{P}{\rightarrow} 0 \quad \text { and } \quad \sqrt{n}\left(\widehat{\beta}_{n}-\beta\right) \stackrel{P}{\rightarrow} 0 .
$$

Note. It appears that a similar result holds when $X \sim \operatorname{Exp}(1 / \lambda)$, but the proof is too long for inclusion here.

\section{Goodness-of-fit tests based on characterizations via mo-} ments of record values. From Corollary 4 (b) we know that $X \sim \operatorname{Exp}(1 / \lambda)$ iff

$$
E\left(Y_{1}^{(k)}\right)^{2}-\frac{2 \lambda}{k} E Y_{2}^{(k)}+\frac{2 \lambda^{2}}{k^{2}}=0 .
$$

Consider the case $\lambda=1$. Then we see that $X \sim \operatorname{Exp}(1)$ iff

$$
E\left(\min \left(X_{1}, \ldots, X_{k}\right)\right)^{2}-\frac{2}{k} E Y_{2}^{(k)}+\frac{2}{k^{2}}=0 .
$$

The idea is to use the sample to obtain an estimate, $\theta_{n}$ say, of the expected value of $\left(Y_{1}^{(k)}\right)^{2}-(2 / k) Y_{2}^{(k)}+2 / k^{2}$ and reject $H$ if $\theta_{n}^{2}$ is large. Since record values are defined in terms of an infinite sequence, it is not clear how one can get estimates of the associated expected values from a finite sample. But they can be estimated indirectly here because when $H$ is true then for each $k$,

$$
E Y_{2}^{(k)}=E X_{1: k}+\frac{1}{k} \quad(\text { cf. [7], [8]), }
$$

and so (3.1) has the form

$$
E\left(X_{1: k}^{2}-\frac{2}{k} X_{1: k}\right)=0 .
$$


Now suppose that $X \sim \operatorname{Exp}(1 / \lambda)$. Since $X \sim \operatorname{Exp}(1 / \lambda) \Leftrightarrow X / \lambda \sim$ $\operatorname{Exp}(1)$, it follows from (3.2) that

$$
E\left(X_{1: k}^{2}-\frac{2 \lambda}{k} X_{1: k}\right)=0 .
$$

Consider first the case $k=1$. Then

$$
E\left(X_{1}^{2}-2 \lambda X_{1}\right)=0 .
$$

The sample $\left(X_{1}, \ldots, X_{n}\right)$ provides an estimator of $E W_{1}$, where $W_{1}=X_{1}^{2}-$ $2 \lambda X_{1}$, of the form

$$
\overline{W_{n}}=\overline{X_{n}^{2}}-2 \lambda \overline{X_{n}},
$$

where $\overline{X_{n}^{2}}=(1 / n) \sum_{j=1}^{n} X_{j}^{2}$. It follows from the CLT that

$$
\sqrt{n} \overline{W_{n}} \stackrel{D}{\rightarrow} W \sim N\left(0, \operatorname{Var}\left(W_{1}\right)\right),
$$

and hence that

$$
T_{n}^{(1)}(\lambda):=n\left(\overline{W_{n}}\right)^{2} / \operatorname{Var}\left(W_{1}\right) \stackrel{D}{\rightarrow} \chi^{2}(1),
$$

which provides a simple asymptotic test of the hypothesis $X \sim \operatorname{Exp}(1 / \lambda)$ when $\lambda$ is specified. Here

$$
\operatorname{Var}\left(W_{1}\right)=E X_{1}^{4}-4 \lambda E X_{1}^{3}+4 \lambda^{2} E X_{1}^{2}=8 \lambda^{4}
$$

since $X_{1} \sim \operatorname{Exp}(1 / \lambda)$ gives $E X_{1}^{m}=m ! \lambda^{m}, m=1,2, \ldots$, and so

$$
T_{n}^{(1)}(\lambda)=\frac{n}{8}\left(\frac{1}{\lambda^{2}} \overline{X_{n}^{2}}-\frac{2}{\lambda} \overline{X_{n}}\right)^{2} .
$$

Thus we have proved

Proposition 3. If $X_{n} \sim \operatorname{Exp}(1 / \lambda), n \geq 1$, are independent then

$$
T_{n}^{(1)}(\lambda)=\frac{n}{8}\left(\frac{1}{\lambda^{2}} \overline{X_{n}^{2}}-\frac{2}{\lambda} \overline{X_{n}}\right)^{2} \stackrel{D}{\rightarrow} \chi^{2}(1) .
$$

Now consider the case $k=2$. Write $U_{1}:=X_{1: 2}=\min \left(X_{1}, X_{2}\right)$. Here from $\left(3.2^{\prime}\right)$ we have to estimate $E W_{1}^{\prime}$, where $W_{1}^{\prime}=U_{1}^{2}-\lambda U_{1}$. The sample $X_{1}, \ldots, X_{2 n}$ provides the sample $W_{1}^{\prime}, \ldots, W_{n}^{\prime}$, where $W_{j}^{\prime}=U_{j}^{2}-\lambda U_{j}$ and $U_{j}=\min \left(X_{2 j-1}, X_{2 j}\right), j=1, \ldots, n$. Then $E W_{1}^{\prime}$ is estimated by

$$
\overline{W_{n}^{\prime}}=\overline{U_{n}^{2}}-\lambda \overline{U_{n}},
$$

and

$$
T_{n}^{(2)}(\lambda):=n\left(\overline{W_{n}^{\prime}}\right)^{2} / \operatorname{Var}\left(W_{1}^{\prime}\right) \stackrel{D}{\rightarrow} \chi^{2}(1) .
$$

Taking into account that $U_{1} \sim \operatorname{Exp}(2 / \lambda)$ we see that $\operatorname{Var}\left(W_{1}^{\prime}\right)=\lambda^{4} / 2$. Thus another simple asymptotic test is provided by 
Proposition 4. If $X_{n} \sim \operatorname{Exp}(1 / \lambda), n \geq 1$, are independent then

$$
T_{n}^{(2)}(\lambda)=\frac{2 n}{\lambda^{4}}\left(\overline{U_{n}^{2}}-\lambda \overline{U_{n}}\right)^{2} \stackrel{D}{\rightarrow} \chi^{2}(1) .
$$

The same argument leads to a similar test for the case $k=3, \ldots, n-1$ based on a sample of size $k n$.

We now consider the case $k=n$. Write $U_{n}=\min \left(X_{1}, \ldots, X_{n}\right)$. Then by $\left(3.2^{\prime}\right)$ we have to estimate $E\left(U_{n}^{2}-(2 \lambda / n) U_{n}\right)$. The obvious estimate is $U_{n}^{2}-(2 \lambda / n) U_{n}$ itself, and then when $\lambda$ is specified the test statistic is

$$
T_{n}^{(n)}(\lambda):=\left(U_{n}^{2}-\frac{2 \lambda}{n} U_{n}\right)^{2} .
$$

As above, under $H, U_{n} \sim \operatorname{Exp}(n / \lambda)$, whence

$$
U:=\frac{n}{\lambda} U_{n} \sim \operatorname{Exp}(1), \quad n \geq 1 .
$$

It follows that

$$
T_{n}^{(n)}(\lambda)=\frac{\lambda^{4}}{n^{4}}\left(U^{2}-2 U\right)^{2}
$$

and so an equivalent test statistic is $T:=\left(U^{2}-2 U\right)^{2}$, which provides an exact test for $H: X \sim \operatorname{Exp}(1 / \lambda)$.

Proposition 5. The significance probability of the test using $T$ is

$$
P_{t}:=P[T>t]= \begin{cases}e^{-1-\sqrt{1+\sqrt{t}}} & \text { if } t>1, \\ e^{-1-\sqrt{1+\sqrt{t}}}+e^{-1+\sqrt{1-\sqrt{t}}}-e^{-1-\sqrt{1-\sqrt{t}}} & \text { if } 0<t<1 .\end{cases}
$$

Proof. The first statement is obtained from the positive root of the equation $u^{2}-2 u-\sqrt{t}=0$, and the second from the positive roots of the equation $\left(u^{2}-2 u\right)^{2}=t$.

In particular we consider the $5 \%$ test of $H$, i.e. $P_{t}=0.05$. But since

$$
P[T>1]=e^{-(1+\sqrt{2})}>0.05
$$

the $5 \%$ test rejects when $U>x_{0}$, where $e^{-x_{0}}=0.05$, i.e. when $x_{0}=3.00$. Thus the exact $5 \%$ test rejects when $(n / \lambda) U_{n}>3$.

We now consider corresponding tests when $\lambda$ is not specified. The general idea is to consider the statistics obtained by replacing $\lambda$ in (3.3) and (3.4) by an estimate $\widehat{\lambda}_{n}$ obtained from the sample.

In this case we have the following results based on $T_{n}^{(1)}(\lambda)$ and $T_{n}^{(2)}(\lambda)$.

Proposition 6. When $F(x)=1-e^{-x / \lambda}, x>0, \lambda>0$, the resulting test statistic is

$$
2 \widehat{T}_{n}^{(1)}:=2 T_{n}^{(1)}\left(\widehat{\lambda}_{n}\right)=\frac{n}{4}\left(\overline{X_{n}^{2}} /\left(\overline{X_{n}}\right)^{2}-2\right)^{2} \stackrel{D}{\rightarrow} \chi^{2}(1),
$$

where $\widehat{\lambda}_{n}=\overline{X_{n}}$. 
Proposition 7. When $F(x)=1-e^{-x / \lambda}, x>0, \lambda>0$, the resulting statistic is

$$
\begin{aligned}
\frac{4}{3} \widehat{T}_{n}^{(2)} & :=\frac{4}{3} T_{n}^{(2)}\left(\widehat{\lambda}_{n}\right)=\frac{8 n}{3 \widehat{\lambda}_{n}^{4}}\left(\overline{U_{n}^{2}}-\widehat{\lambda}_{n} \overline{U_{n}}\right)^{2} \\
& =\frac{8 n}{3}\left(\frac{\overline{U_{n}^{2}}}{\left(\overline{X_{2 n}}\right)^{2}}-\frac{\overline{U_{n}}}{\overline{X_{2 n}}}\right)^{2} \stackrel{D}{\rightarrow} \chi^{2}(1),
\end{aligned}
$$

where $\widehat{\lambda}_{n}=\overline{X_{2 n}}$.

Proof of Proposition 6. Consider $\mathbf{V}=\left(\begin{array}{c}X^{2} \\ X\end{array}\right)$. Then $\overline{\mathbf{V}_{n}}=\left(\overline{X_{n}^{2}}\right)$ and by the CLT,

$$
\sqrt{n}\left(\overline{\mathbf{V}_{n}}-\mu\right) \stackrel{D}{\rightarrow} N(0, \Sigma),
$$

where

$$
\mu=E \mathbf{V}=\left(\begin{array}{c}
2 \lambda^{2} \\
\lambda
\end{array}\right) \quad \text { and } \quad \Sigma=\operatorname{Var}(\mathbf{V})=\left(\begin{array}{cc}
20 \lambda^{4} & 4 \lambda^{3} \\
4 \lambda^{3} & \lambda^{2}
\end{array}\right) .
$$

We now use a theorem on asymptotic distributions of functions of statistics (cf. [10], p. 260), with $g(\mathbf{x})=x_{1} / x_{2}^{2}$. Then

$$
g(\mu)=2, \quad \gamma:=\left(\frac{\partial g}{\partial \mathbf{x}}\right)_{\mathbf{x}=\mu}=\left(\begin{array}{c}
1 / \lambda^{2} \\
-4 / \lambda
\end{array}\right), \quad \gamma^{\prime} \Sigma \gamma=4
$$

and so

$$
\sqrt{n}\left(g\left(\overline{\mathbf{V}_{n}}\right)-g(\mu)\right)=\sqrt{n}\left(\overline{X_{n}^{2}} /\left(\overline{X_{n}}\right)^{2}-2\right) \stackrel{D}{\rightarrow} W \sim N(0,4),
$$

and

$$
2 \widehat{T}_{n}^{(1)}=\frac{n}{4}\left(\overline{X_{n}^{2}} /\left(\overline{X_{n}}\right)^{2}-2\right)^{2} \stackrel{D}{\rightarrow} \chi^{2}(1) .
$$

In the proof of Proposition 7 we shall apply the following

LEMma 2. Let $X_{1} \sim \operatorname{Exp}(1 / \lambda), X_{2} \sim \operatorname{Exp}(1 / \lambda)$ be independent and put $U:=U_{1}=\min \left(X_{1}, X_{2}\right), Y=\left(X_{1}+X_{2}\right) / 2$. Then the pdf of $U$ and $Y$ is

$$
h(u, y)=\frac{4}{\lambda^{2}} e^{-2 y / \lambda}, \quad 0<u<y, y>0,
$$

and

$$
\operatorname{Cov}(U, Y)=\lambda^{2} / 4, \quad \operatorname{Cov}\left(U^{2}, Y\right)=\lambda^{3} / 2 .
$$

Proof of Proposition \%. We now consider

$$
\mathbf{V}=\left(\begin{array}{c}
U_{1}^{2} \\
U_{1} \\
\left(X_{1}+X_{2}\right) / 2
\end{array}\right), \quad \overline{\mathbf{V}_{n}}=\left(\begin{array}{c}
\overline{U_{n}^{2}} \\
\overline{U_{n}} \\
\overline{X_{2 n}}
\end{array}\right)
$$

By the CLT,

$$
\sqrt{n}\left(\overline{\mathbf{V}_{n}}-\mu\right) \stackrel{D}{\rightarrow} N(\mathbf{0}, \Sigma),
$$


where $\mu=E \mathbf{V}$ and $\Sigma=\operatorname{Var} \mathbf{V}$. Now using Lemma 2 we get

$$
\mu=\left(\begin{array}{c}
\lambda^{2} / 2 \\
\lambda / 2 \\
\lambda
\end{array}\right) \quad \text { and } \quad \Sigma=\left(\begin{array}{ccc}
5 \lambda^{4} / 4 & \lambda^{3} / 2 & \lambda^{3} / 2 \\
\lambda^{3} / 2 & \lambda^{2} / 4 & \lambda^{2} / 4 \\
\lambda^{3} / 2 & \lambda^{2} / 4 & \lambda^{2} / 2
\end{array}\right)
$$

Using the above theorem of Wilks [10] with $g(\mathbf{x})=x_{1} / x_{3}^{2}-x_{2} / x_{3}$ we have

$$
g(\mu)=0, \quad \gamma:=\left(\frac{\partial g}{\partial \mathbf{x}}\right)_{\mathbf{x}=\mu}=\left(\begin{array}{c}
1 / \lambda^{2} \\
-1 / \lambda \\
-1 / 2 \lambda
\end{array}\right), \quad \gamma^{\prime} \Sigma \gamma=3 / 8
$$

Thus

$$
\sqrt{n}\left(\overline{U_{n}^{2}} /\left(\overline{X_{2 n}}\right)^{2}-\overline{U_{n}} / \overline{X_{2 n}}\right) \stackrel{D}{\rightarrow} N(0,3 / 8)
$$

and so

$$
\frac{8 n}{3}\left(\overline{U_{n}^{2}} /\left(\overline{X_{2 n}}\right)^{2}-\overline{U_{n}} / \overline{X_{2 n}}\right)^{2}=\frac{4}{3} \widehat{T}_{n}^{(2)} \stackrel{D}{\rightarrow} \chi^{2}(1) .
$$

REMARK. Instead of the MLE $\widehat{\lambda}_{n}$ one could consider the corresponding estimate $\lambda_{n}^{*}:=2 \overline{U_{n}}$ obtained from $U_{1}, \ldots, U_{n}$. But since $\operatorname{Var}\left(\lambda_{n}^{*}\right)>$ $\operatorname{Var}\left(\widehat{\lambda}_{n}\right)$, one would expect intuitively that the resulting test would be in some respect poorer. This leads to

$$
T_{n}^{*(2)}:=\frac{n}{8}\left(\overline{U_{n}^{2}} /\left(\overline{U_{n}}\right)^{2}-2\right)^{2} .
$$

Then it follows as in the discussion of Proposition 6 that

$$
2 T_{n}^{*(2)} \stackrel{D}{\rightarrow} \chi^{2}(1) .
$$

Referring to (3.5), in the case when $k=n$ we use the statistic $\widehat{U}_{n}=$ $n U_{n} / \widehat{\lambda}_{n}$ where $\widehat{\lambda}_{n}=\bar{X}_{n}$. Consider the test that rejects when $\widehat{U}_{n}>3$. Now $\widehat{U}_{n}=\left(\lambda / \widehat{\lambda}_{n}\right) U$ where $U=n U_{n} / \lambda \sim \operatorname{Exp}(1)$, and $\widehat{U}_{n} \stackrel{D}{\rightarrow} U$ since $\widehat{\lambda}_{n} \stackrel{P}{\rightarrow} \lambda$. Thus

$$
\lim _{n \rightarrow \infty} P\left(\widehat{U}_{n}>3\right)=P(U>3)=0.05
$$

and so this is an asymptotic $5 \%$ test.

Moreover, we have

Proposition 8. Let $\widehat{T}_{n}:=\left(\widehat{U}_{n}^{2}-2 \widehat{U}_{n}\right)^{2}$ and let $\widehat{P}_{t}:=P\left[\widehat{T}_{n}>t\right]$ stand for the associated significance probability. Then $\lim _{n \rightarrow \infty} \widehat{P}_{t}=P_{t}$, where $P_{t}$ is given by Proposition 5.

P r o of. Since $\widehat{U}_{n}=\left(\lambda / \widehat{\lambda}_{n}\right) U$, we have

$$
\widehat{T}_{n}=\left[\left(\lambda / \widehat{\lambda}_{n}\right)^{2} U^{2}-2\left(\lambda / \widehat{\lambda}_{n}\right) U\right]^{2} \stackrel{D}{\rightarrow} T,
$$

which ends the proof. 
4. Simulations. Here we consider tests of $\operatorname{Exp}(1 / \lambda)$.

First note that some goodness-of-fit tests based on a characterization were also proposed in [6] where the $\chi^{2}(2)$ approximation was used. Here we observe that a long and complicated argument shows that

$$
\begin{aligned}
D_{n}\left(\widehat{\lambda}_{n}\right)= & 45 n\left[\frac{110}{19}\left(\frac{1}{2 n} \sum_{j=1}^{2 n}\left(1-\exp \left(-X_{j} / \widehat{\lambda}_{n}\right)\right)^{2}-\frac{1}{3}\right)^{2}\right. \\
& +2\left(\frac{1}{n} \sum_{j=1}^{n} \exp \left(-\left(X_{2 j}+X_{2 j-1}+\left|X_{2 j}-X_{2 j-1}\right|\right) /\left(2 \widehat{\lambda}_{n}\right)\right)-\frac{1}{3}\right)^{2} \\
& -4\left(\frac{1}{2 n} \sum_{j=1}^{2 n}\left(1-\exp \left(-X_{j} / \widehat{\lambda}_{n}\right)\right)^{2}-\frac{1}{3}\right) \\
& \left.\times\left(\frac{1}{n} \sum_{j=1}^{n} \exp \left(-\left(X_{2 j}+X_{2 j-1}+\left|X_{2 j}-X_{2 j-1}\right|\right) /\left(2 \widehat{\lambda}_{n}\right)\right)-\frac{1}{3}\right)\right] \\
\rightarrow & \chi^{2}(2), \quad \text { where } \widehat{\lambda}_{n}=\bar{X}_{2 n} .
\end{aligned}
$$

Simulation strongly confirms that indeed $D_{n}\left(\widehat{\lambda}_{n}\right) \stackrel{D}{\rightarrow} \chi^{2}(2)$, and so $D_{n}\left(\widehat{\lambda}_{n}\right)$ provides a simple test for $X \sim \operatorname{Exp}(1 / \lambda)$. We see that $D_{n}\left(\widehat{\lambda}_{n}\right)$ differs from $D_{n}$ of [6] by having leading coefficient $\frac{110}{19}$ instead of $\frac{5}{2}$.

The test statistics investigated here are: $D_{n}\left(\widehat{\lambda}_{n}\right)$,

$$
\begin{aligned}
V_{n}^{2}\left(\widehat{\lambda}_{n}\right)= & 90 n\left[\frac{1}{n} \sum_{j=1}^{n}\left(1-\exp \left(-\left(X_{2 j}+X_{2 j-1}+\left|X_{2 j}-X_{2 j-1}\right|\right) /\left(2 \widehat{\lambda}_{n}\right)\right)\right)\right. \\
& \left.-\frac{1}{2 n} \sum_{j=1}^{2 n}\left(1-\exp \left(X_{j} / \widehat{\lambda}_{n}\right)\right)^{2}-\frac{1}{3}\right]^{2},
\end{aligned}
$$

$\widehat{T}_{n}^{(1)}, \widehat{T}_{n}^{(2)}, T_{n}^{*(2)}$ and $\widehat{T}_{n}$ from Propositions 8 and 5 .

Firstly, 2000 samples of size 20 were obtained from an exponential distribution and the 6 statistics evaluated for each sample, and tested for significance at the $10 \%, 5 \%$ and $1 \%$ levels approximately. For $D_{n}\left(\widehat{\lambda}_{n}\right)$ the $\chi^{2}(2)$ approximation was used, so that for the approximate $10 \%$ test the observed value is significant if it exceeds 4.605 etc. Then for $V_{n}^{2}\left(\widehat{\lambda}_{n}\right), \widehat{T}_{n}^{(1)}, \widehat{T}_{n}^{(2)}$ and $T_{n}^{*(2)}$ the $\chi^{2}(1)$ approximation was used, and for $\widehat{T}_{n}$ the approximation obtained from Propositions 5 and 8. In each case the percentage of significant samples is shown in the table below. Then this was repeated for samples of size 40, 100, 200. 


\begin{tabular}{rrcccccr}
\hline & $n$ & $D_{n}\left(\widehat{\lambda}_{n}\right)$ & $V_{n}^{2}\left(\widehat{\lambda}_{n}\right)$ & $\widehat{T}_{n}^{(1)}$ & $\widehat{T}_{n}^{(2)}$ & $T_{n}^{*(2)}$ & $\widehat{T}_{n}$ \\
\hline \multirow{4}{*}{$10 \%$} & 20 & 8.1 & 11.7 & 3.2 & 3.6 & 1.4 & 10.0 \\
& 40 & 8.6 & 9.6 & 5.8 & 4.5 & 3.8 & 10.8 \\
& 100 & 9.6 & 8.7 & 7.8 & 6.5 & 5.5 & 9.6 \\
& 200 & 9.3 & 9.4 & 8.9 & 7.9 & 7.9 & 10.5 \\
\hline \multirow{4}{*}{$5 \%$} & 20 & 3.6 & 5.7 & 2.0 & 2.5 & 0.8 & 4.6 \\
& 40 & 4.3 & 4.8 & 3.5 & 3.1 & 2.5 & 5.5 \\
& 100 & 5.0 & 4.1 & 3.8 & 3.8 & 2.9 & 5.3 \\
& 200 & 4.2 & 4.3 & 3.9 & 4.5 & 4.1 & 5.5 \\
\hline & 20 & 0.8 & 0.9 & 0.7 & 0.8 & 0.4 & 1.0 \\
$1 \%$ & 40 & 0.7 & 1.1 & 1.4 & 1.8 & 1.0 & 0.7 \\
& 100 & 1.2 & 0.9 & 1.4 & 1.7 & 1.3 & 1.3 \\
& 200 & 0.7 & 0.9 & 0.8 & 2.1 & 1.8 & 1.2 \\
\hline
\end{tabular}

It appears that $\widehat{T}_{n}$ performs the best, followed by $D_{n}\left(\widehat{\lambda}_{n}\right)$ and $V_{n}^{2}\left(\widehat{\lambda}_{n}\right)$, and the other tests are poorer when $n$ is small.

REMARK. The above statistics $D_{n}\left(\widehat{\lambda}_{n}\right)$ and $V_{n}^{2}\left(\widehat{\lambda}_{n}\right)$ are derived from the statistics

$$
\begin{aligned}
D_{n} & =45 n\left[\frac{110}{19}\left(\bar{Y}_{n}-\frac{1}{3}\right)^{2}+2\left(\bar{Z}_{n}-\frac{2}{3}\right)^{2}-4\left(\bar{Y}_{n}-\frac{1}{3}\right)\left(\bar{Z}_{n}-\frac{2}{3}\right)\right], \\
V_{n}^{2} & =90 n\left(\bar{Z}_{n}-\frac{1}{2} \bar{Y}_{n}-\frac{1}{3}\right)^{2},
\end{aligned}
$$

respectively.

\section{References}

[1] W. Dziubdziela and B. Kopociński, Limiting properties of the $k$-th record values, Zastos. Mat. 15 (1976), 187-190.

[2] Z. Grudzień and D. Szynal, Characterization of continuous distributions in terms of moments of extremal statistics, J. Math. Sci. 81 (1996), 2912-2936.

[3] - - - Characterizations of continuous distributions via moments of the $k$-th record values with random indices, Brandenburgische Technische Universität Cottbus, Fakultät für Mathematik, Naturwissenschaften und Informatik, Reihe Mathematik, M-05/1997 (1997).

[4] - - - Characterizations of continuous distributions via moments of record values, J. Appl. Statist. Sci. 9 (2000), 93-104.

[5] G. D. Lin, Characterizations of continuous distributions via expected values of two functions of order statistics, Sankhyā Ser. A 52 (1990), 84-90.

[6] K. Morris and D. Szynal, A goodness-of-fit test for the uniform distribution based on a characterization, in: XX Internat. Sympos. on Stability Problems for Stochastic Models (Lublin-Nałęczów, 1999), Abstracts, p. 119, submitted to J. Math. Sci.

[7] P. Pawlas and D. Szynal, Relations for single and product moments of $k$-th record values from exponential and Gumbel distributions, J. Appl. Statist. Sci. 7 (1998), $53-62$. 
[8] P. Pawlas and D. Szynal, Recurrence relations for single and product moments of $k$-th record values from Weibull distributions, and a characterization, ibid. 10 (2000), 17-26.

[9] Y. H. Too and G. D. Lin, Characterizations of uniform and exponential distributions, Statist. Probab. Lett. 7 (1989), 357-359.

[10] S. S. Wilks, Mathematical Statistics, Wiley, New York, 1962.

Kerwin Morris

Department of Statistics

University of Adelaide

North Tce, Adelaide

South Australia, 5001

E-mail: kmorris@stats.adelaide.edu.au
Dominik Szynal

Institute of Mathematics

Maria Curie-Skłodowska University

Pl. M. Curie-Skłodowskiej 1

20-031 Lublin, Poland

E-mail: szynal@golem.umcs.lublin.pl

Received on 7.2.2000;

revised version on 2.6.2000 